International Journal of Pure and Applied Mathematics

Volume 95 No. 4 2014, 611-622

ISSN: 1311-8080 (printed version); ISSN: 1314-3395 (on-line version)

url: http://www.ijpam.eu

doi: http://dx.doi.org/10.12732/ijpam.v95i4.14

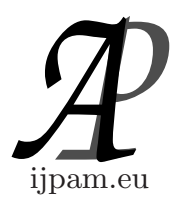

\title{
STRONGLY SEMICOMMUTATIVE RINGS RELATIVE TO A MONOID
}

\author{
Ayoub Elshokry ${ }^{1}$, Eltiyeb Ali $^{2}$ \\ ${ }^{1,2}$ Department of Mathematics \\ Northwest Normal University \\ Lanzhou 730070, P.R. CHINA \\ ${ }^{1,2}$ Department of Mathematics \\ University of Khartoum \\ Omdurman, SUDAN
}

\begin{abstract}
For a monoid $M$, we introduce strongly semicommutative rings relative to $M$, which are a generalization of strongly semicommutative rings, and investigates its properties. We show that every reduced ring is strongly $M$-semicommutative for any unique product monoid $M$. Also it is shown that for a monoid $M$ and an ideal $I$ of $R$. If $I$ is a reduced ring and $R / I$ is strongly $M$-semicommutative, then $R$ is strongly $M$-semicommutative.
\end{abstract}

AMS Subject Classification: 16S36, 16N60, 16U99

Key Words: unique product monoid, reduced rings, semicommutative rings, strongly semicommutative rings, strongly semicommutative rings relative to $M$

\section{Introduction}

Throughout this article, $R$ and $M$ denote an associative ring with identity and a monoid, respectively. Recall that a ring is reduced if it has no nonzero nilpotent elements. Lambek [9] called a ring $R$ symmetric provided $a b c=0$ implies

Received: June 30, 2014

(C) 2014 Academic Publications, Ltd.

$\S_{\text {Correspondence author }}$ url: www.acadpubl.eu 
$a c b=0$ for $a, b, c \in R$. Habeb [10] called a $\operatorname{ring} R$ zero commutative if $R$ satisfies the condition: $a b=0$ implies $b a=0$ for $a, b \in R$, while Cohn [14] used the term reversible for what is called zero commutative. A generalization of a reversible ring is a semicommutative ring. A ring $R$ is semicommutative if $a b=0$ implies $a R b=0$ for $a, b \in R$. Historically, some of the earliest results known to us about semicommutative rings (although not so called at the time) was due to Shin [6]. He proved that (i) $R$ is semicommutative if and only if $r_{R}(a)$ is an ideal of $R$ where $r_{R}(a)=\{b \in R \mid a b=0\}$ [6, Lemma 1.2], (ii) every reduced ring is symmetric [6, Lemma 1.1] (but the converse does not hold [3, Example II.5]), and (iii) any symmetric ring is semicommutative but the converse does not hold ([6, Proposition 1.4 and Example 5.4(a)]). Semicommutative rings were also studied under the name zero insertive by Habeb [10]. In [12], Kim and Lee showed that polynomial rings over reversible rings need not be reversible. In [7], Yang and Liu introduced the notation of strongly reversible. A ring $\mathrm{R}$ is called strongly reversible, if whenever polynomials $f(x), g(x) \in R[x]$ satisfy $f(x) g(x)=0$ implies $g(x) f(x)=0$. All reduced rings are strongly reversible but converse is not true. Another generalization of a reduced ring is an Armendariz ring. Rege and Chhawchharia [11] called a ring $R$ Armendariz if whenever any polynomials $f(x)=a_{0}+a_{1} x+a_{2} x^{2}+\cdots+a_{n} x^{n}, g(x)=b_{0}+b_{1} x+b_{2} x^{2}+\cdots+$ $b_{m} x^{m} \in R[x]$ satisfy $f(x) g(x)=0$, then $a_{i} b_{j}=0$ for each $i, j$. (The converse is always true). In [19], Z. Liu studied a generalization of Armendariz rings, which is called $M$-Armendariz rings, where $M$ is monoid. A ring $R$ is called $M$-Armendariz if whenever $\alpha=a_{1} g_{1}+a_{2} g_{2}+\cdots+a_{n} g_{n}, \beta=b_{1} h_{1}+b_{2} h_{2}+$ $\cdots+b_{m} g_{m} \in R[M]$, satisfy $\alpha \beta=0$, then $a_{i} b_{j}=0$, for each $i, j$, where $g_{i}, h_{j} \in$ $M$. In [1], a ring $R$ is called strongly $M$-reversible, whenever $\alpha \beta=0$ implies $\beta \alpha=0$, where $\alpha, \beta \in R[M]$. Properties, examples and counterexamples of semicommutative rings were given in Huh, Lee and Smoktunowicz [2], Kim and Lee [12], Liu [20] and Yang [8]. In [18, Corollary 2.3], it was claimed that all semicommutative rings are McCoy. However, Hiranos claim assumed that if $R$ is semicommutative then $R[x]$ is semicommutative, but this was later shown to be false in [2, Example 2]. Moreover, Nielsen [15], gave an example to show that a semicommutative ring $R$ need not be right McCoy, also he proved that the polynomial ring $R[x]$ over it actually is not semicommutative. In [17], a ring $R$ is called strongly semicommutative if whenever polynomials $f(x), g(x)$ in $R[x]$ satisfy $f(x) g(x)=0$, then $f(x) R[x] g(x)=0$.

Recall that a monoid $M$ is called a u.p.-monoid (unique product monoid) if for any two nonempty finite subsets $A, B \subseteq M$ there exists an element $g \in M$ uniquely in the form $a b$ where $a \in A$ and $b \in B$. The class of u.p.-monoid is quite large and important (see $[5,4]$ ). For example, this class includes the right 
or left ordered monoids, submonoids of a free group, and torsion-free nilpotent groups. Every u.p.-monoid $M$ has no nonunity element of finite order.

Motivated by the results of Z. Liu [19], Y. Gang and D.R. Juan [17] and T. K. Kwak, Y. Lee and S. J. Yun [16], we investigate a generalization of strongly semicommutative rings which we call strongly M-semicommutative rings.

\section{Main Results}

For a monid $M$, e will always stand for the identity of $M$. If $R$ is a ring, then $R[M]$ denotes the monoid ring over $R$. In this article we introduce the concept of a strongly $M$-semicommutative ring and investigate its properties. We start with the following definition.

Definition 2.1. A ring $R$ is called strongly $M$-semicommutative, if whenever elements $\alpha=a_{1} g_{1}+a_{2} g_{2}+\cdots+a_{n} g_{n}, \beta=b_{1} h_{1}+b_{2} h_{2}+\cdots+b_{m} h_{m} \in R[M]$, with $g_{i}, h_{j} \in M$ satisfy $\alpha \beta=0$, then $\alpha R[M] \beta=0$.

Let $M=(N \cup\{0\},+)$. Then a ring $R$ is strongly $M$-semicommutative if and only if $R$ is strongly semicommutative.

Lemma 2.2. [1, Lemma 1]. Let $M$ be a u.p.-monoid and $R$ a reduced, then $R[M]$ is reduced.

Lemma 2.3. [16, Remark 2.4(3)]. Strongly reversible rings are clearly strongly semicommutative, but the converse does not hold.

Proposition 2.4. Let $M$ be a u.p.-monoid and $R$ a reduced ring. If $R$ is strongly $M$-reversible ring then $R$ is strongly $M$-semicommutative.

Proof. Suppose $\alpha=a_{1} g_{1}+\cdots+a_{n} g_{n}$ and $\beta=b_{1} h_{1}+\cdots+b_{m} h_{m} \in R[M]$, with $a_{i}, b_{j} \in R$ and $g_{i}, h_{j} \in M$ for all $i, j$. Let $\alpha \beta=0$. Then $\beta \alpha=0$, since $R$ is strongly $M$-reversible and $\beta \alpha \gamma=0$ for any $\gamma \in R[M]$, so $\alpha \gamma \beta=0$, since $R[M]$ is reduced by Lemma 2.2. Hence $R$ is strongly $M$-semicommutative.

Proposition 2.5. Let $M$ be a u.p.-monoid and $R$ a reduced. Then $R$ is strongly $M$-semicommutative.

Proof. Let $M$ be a u.p.-monoid and $R$ a reduced ring. Then by [1, Proposion 1], $R$ is strongly $M$-reversible. Thus, $R$ is strongly $M$-semicommutative by Proposition 2.4.

Let $(M, \leq)$ be an ordered monoid. If for any $g, g^{\prime}, h \in M, g<g^{\prime}$ implies that $g h<g^{\prime} h$ and $h g<h g^{\prime}$, then $(M, \leq)$ is called a strictly ordered monoid. 
Corollary 2.6. Let $M$ be strictly totally ordered monoid and $R$ a reduced ring. Then $R$ is strongly $M$-semicommutative.

Lemma 2.7. The class of strongly $M$-semicommutative is closed under subrings and direct products.

Proposition 2.8. Let $M$ be a commutative, cancellative monoid and $N$ and ideal of $M$. If $R$ is strongly $N$-semicommutative ring, then $R$ is strongly $M$-semicommutative ring.

Proof. Suppose that $\alpha=a_{1} g_{1}+a_{2} g_{2}+\cdots+a_{n} g_{n}$ and $\beta=b_{1} h_{1}+b_{2} h_{2}+\cdots+$ $b_{m} h_{m} \in R[M]$, such that $\alpha \beta=0$. Take $g \in N$, then $g g_{1}, g g_{2}, \cdots, g g_{n}, h_{1} g, h_{2} g, \cdots$ ,$h_{m} g \in N$ and $g g_{i} \neq g g_{j}$ and $h_{i} g \neq h_{j} g$ when $i \neq j$. So

$$
\alpha_{1} \beta_{1}=\left(\sum_{i=1}^{n} a_{i} g g_{i}\right)\left(\sum_{j=1}^{m} b_{j} h_{j} g\right)=0 .
$$

Since $R$ is strongly $N$-semicommutative so $\alpha_{1} \gamma_{1} \beta_{1}=0$ for any $\gamma_{1} \in R[N]$. Thus, $\alpha \gamma \beta=0$, for any $\gamma \in R[M]$. Therefore $R$ is strongly $M$-semicommutative.

Theorem 2.9. For a monoid $M$. Suppose that $R / I$ is strongly $M$ semicommutative for some ideal $I$ of a ring $R$. If $I$ is a reduced ring then $R$ is strongly $M$-semicommutative.

Proof. Let $\alpha \beta=0$ with $\alpha, \beta \in R[M]$. Then we have $\alpha R \beta \subseteq I[M]$ and $\beta I \alpha=0$, since $\beta I \alpha \subseteq I[M],(\beta I \alpha)^{2}=0$ and $I[M]$ is reduced. Thus, $(\alpha R \beta I)^{2}=$ $\alpha R \beta I \alpha R \beta I=0$ and so $\alpha R \beta I=0$. Hence $(\alpha R \beta)^{2} \subseteq \alpha R \beta I=0$ since $\alpha R \beta \subseteq$ $I[M]$. Then $\alpha R \beta=0$ since $I[M]$ is reduced. Therefore $R$ is strongly $M$ semicommutative.

Recall that an element $u$ of a ring $R$ is right regular if $u r=0$ implies $r=0$ for $r \in R$. Similarly, left regular elements can be defined. An element is regular if it is both left and right regular (and hence not a zero divisor).

Proposition 2.10. Let $R$ be a ring and $\Delta$ be a multiplicative monoid in $R$ consisting of central regular elements. Then $R$ is strongly $M$-semicommutative if and only if so is $\Delta^{-1} R$.

Proof. $(\Leftarrow)$ This is obvious since $R$ is a subring of $\Delta^{-1} R$.

$(\Rightarrow)$ Suppose that $R$ is strongly $M$-semicommutative. Let $\phi \psi=0$, for $\phi=u^{-1} \alpha$ and $\psi=v^{-1} \beta \in\left(\Delta^{-1} R\right)[M]$ where $u, v$ are regular and $\alpha, \beta \in$ $R[M]$. Since $\Delta$ is contained in the center of $R$ then we have $0=\phi \psi=$ 
$u^{-1} \alpha v^{-1} \beta=\left(u^{-1} v^{-1}\right) \alpha \beta=(u v)^{-1} \alpha \beta$ and so $\alpha \beta=0$. Since $R$ is strongly $M$ semicommutative, $\alpha R \beta=0$ and $\alpha s^{-1} R \beta=0$ for any regular element $s$. This implies $\phi \Delta^{-1} R \beta=0$ and therefore $\Delta^{-1} R$ is strongly $M$-semicommutative.

The ring of Laurent polynomials in $x$, with coefficients in a ring $R$, consists of all formal sum $\sum_{i=k}^{n} m_{i} x^{i}$ with obvious addition and multiplication, where $m_{i} \in R$ and $k, n$ are (possibly negative) integers, denote it by $R\left[x ; x^{-1}\right]$.

Corollary 2.11. Let $M$ be a monoid, for a ring $R$. $R[x]$ is strongly $M$ semicommutative if and only if $R\left[x ; x^{-1}\right]$ is strongly $M$-semicommutative.

Proposition 2.12. Let $M$ be a monoid, and $R$ be a ring, e central idempotent of $R$. Then the following statements are equivalent:

1. $R$ is strongly $M$-semicommutative.

2. $e R$ and $(1-e) R$ are strongly $M$-semicommutative.

Proof. $(1) \Leftrightarrow(2)$ This is straightforward since subrings and finite direct products of strongly $M$-semicommutative rings are strongly $M$-semicommutative by Lemma 2.7 .

Rege-Chhawchhaaria showed that commutative (hence semicommutative) rings need not to be Armendariz in [11, Example 3.2]. Conversely Huh, Lee and Smoktunowicz [2], gave a ring which is Armendariz but not semicommutative. However we have the following result.

Proposition 2.13. For a monoid $M$. Let $R$ be an $M$-Armendariz. If $R$ is a semicommutative ring, then $R$ is strongly $M$-semicommutative.

Proof. Suppose that $\alpha=\sum_{i=1}^{n} a_{i} g_{i}, \beta=\sum_{j=1}^{m} b_{j} h_{j} \in R[M]$ satisfy $\alpha \beta=0$. Then since $R$ is $M$-Armendariz, each $a_{i} b_{j}$ is zero, additionally $R$ is semicommutative, therefore $a_{i} r b_{j}=0$ for any element $r$ in $R$ for all $i, j$. Now it is easy to check that $\alpha \gamma \beta=0$ for any $\gamma \in R[M]$.

Since reversible rings are semicommutative, the following corollary is clear.

Corollary 2.14. Let $M$ be a monoid and $R$ be an $M$-Armendariz ring. If $R$ is a reversible ring, then $R$ is strongly $M$-semicommutative ring.

Lemma 2.15. Let $M$ be a strictly totally ordered monoid and $R$ a reduced ring. Then $R[M]$ is reduced. 
Proof. Suppose that $\alpha=a_{1} g_{1}+\cdots+a_{n} g_{n} \in R[M]$ is such that $\alpha^{2}=0$. Not loss the generality we assume that $g_{1}<g_{2}<\cdots<g_{n}$. Then from $\alpha^{2}=0$ it follows that

$$
a_{1}^{2} g_{1}^{2}+a_{1} a_{2} g_{1} g_{2}+a_{2} a_{1} g_{2} g_{1}+\cdots+a_{n}^{2} g_{n} g_{n}=0 .
$$

Suppose $g_{1} g_{1}=g_{i} g_{j}$ for some $1 \leq i, j \leq n$. Then $g_{1} \leq g_{i}, g_{1} \leq g_{j}$. If $g_{1}<g_{i}$, then $g_{1} g_{1}<g_{i} g_{1} \leq g_{i} g_{j}=g_{1} g_{1}$, a contradiction. Thus, $g_{1}=g_{i}$. Similarly $g_{1}=g_{j}$. Thus, we have $a_{1}^{2}=0$ and so $a_{1}=0$ since $R$ is reduced. Now $\alpha=a_{2} g_{2}+\cdots+a_{n} g_{n}$. By analogy with above proof, we have $a_{2}=0, \cdots$, $a_{n}=0$. Thus $\alpha=0$. This means that $R[M]$ is reduced.

Lemma 2.16. [19, Corollary 1.2] Let $M$ be a strictly totally ordered monoid and $R$ a reduced ring. Then $R$ is $M$-Armendariz.

Proposition 2.17. Let $M$ be a strictly totally ordered monoid and $R$ a reduced ring. If $R$ is strongly $M$-smeicommutative, then $S_{3}(R)$ is strongly $M$-smeicommutative.

Proof. For $\left(\begin{array}{ccc}a_{1} & b_{1} & c_{1} \\ 0 & a_{1} & d_{1} \\ 0 & 0 & a_{1}\end{array}\right),\left(\begin{array}{ccc}a_{2} & b_{2} & c_{2} \\ 0 & a_{2} & d_{2} \\ 0 & 0 & a_{2}\end{array}\right) \in S_{3}(R)$.

We complete the proof by adapting the proof of [13, proposition 2] or [12, proposition 1.2]. It is easy to see that there exists an isomorphism of rings $S_{3}(R)[M] \rightarrow S_{3}(R[M])$ define by:

$$
\sum_{i=1}^{n}\left(\begin{array}{ccc}
a_{i} & b_{i} & c_{i} \\
0 & a_{i} & d_{i} \\
0 & 0 & a_{i}
\end{array}\right)^{g_{i}} \rightarrow\left(\begin{array}{ccc}
\sum_{i=1}^{n} a_{i} g_{i} & \sum_{i=1}^{n} b_{i} g_{i} & \sum_{i=1}^{n} c_{i} g_{i} \\
0 & \sum_{i=1}^{n} a_{i} g_{i} & \sum_{i=1}^{n} d_{i} g_{i} \\
0 & 0 & \sum_{i=1}^{n} a_{i} g_{i}
\end{array}\right)
$$

Suppose that $\alpha=A_{1} g_{1}+A_{2} g_{2}+\cdots+A_{n} g_{n}$, and $\beta=B_{1} h_{1}+B_{2} h_{2}+\cdots+$ $B_{m} h_{m} \in S_{3}(R)[M]$ are such that $\alpha \beta=0$, where $A_{i}, B_{j} \in S_{3}(R)$. We claim $\alpha S_{3}(R)[M] \beta=0$ for each $i, j$. Assume that

$$
A_{i}=\left(\begin{array}{ccc}
a_{i} & b_{i} & c_{i} \\
0 & a_{i} & d_{i} \\
0 & 0 & a_{i}
\end{array}\right), B_{j}=\left(\begin{array}{ccc}
a_{j}^{\prime} & b_{j}^{\prime} & c_{j}^{\prime} \\
0 & a_{j} & d_{j} \\
0 & 0 & a_{j}
\end{array}\right)^{.}
$$

Then we have

$$
\left(\begin{array}{ccc}
\sum_{i=1}^{n} a_{i} g_{i} & \sum_{i=1}^{n} b_{i} g_{i} & \sum_{i=1}^{n} c_{i} g_{i} \\
0 & \sum_{i=1}^{n} a_{i} g_{i} & \sum_{i=1}^{n} d_{i} g_{i} \\
0 & 0 & \sum_{i=1}^{n} a_{i} g_{i}
\end{array}\right)
$$




$$
\times\left(\begin{array}{ccc}
\sum_{j=1}^{m} a_{j}^{\prime} h_{j} & \sum_{j=1}^{m} b_{j}^{\prime} h_{j} & \sum_{j=1}^{m} c_{j}^{\prime} h_{j} \\
0 & \sum_{j=1}^{m} a_{j} h_{j} & \sum_{j=1}^{m} d_{j} h_{j} \\
0 & 0 & \sum_{j=1}^{m} a_{j} h_{j}
\end{array}\right)=0
$$

Thus

$$
\begin{gathered}
\left(\sum_{i=1}^{n} a_{i} g_{i}\right)\left(\sum_{j=1}^{m} a_{j}^{\prime} h_{j}\right)=0, \\
\left(\sum_{i=1}^{n} a_{i} g_{i}\right)\left(\sum_{j=1}^{m} b_{j}^{\prime} h_{j}\right)+\left(\sum_{i=1}^{n} b_{i} g_{i}\right)\left(\sum_{j=1}^{m} a_{j}^{\prime} h_{j}\right)=0, \\
\left(\sum_{i=1}^{n} a_{i} g_{i}\right)\left(\sum_{j=1}^{m} c_{j}^{\prime} h_{j}\right)+\left(\sum_{i=1}^{n} b_{i} g_{i}\right)\left(\sum_{j=1}^{m} \dot{d}_{j}^{\prime} h_{j}\right)+\left(\sum_{i=1}^{n} c_{i} g_{i}\right)\left(\sum_{j=1}^{m} a_{j}^{\prime} h_{j}\right)=0, \\
\left(\sum_{i=1}^{n} a_{i} g_{i}\right)\left(\sum_{j=1}^{m} d_{j}^{\prime} h_{j}\right)+\left(\sum_{i=1}^{n} d_{i} g_{i}\right)\left(\sum_{j=1}^{m} a_{j}^{\prime} h_{j}\right)=0 .
\end{gathered}
$$

By Lemma 2.16 $R$ is $M$-Armendariz, from Eq. (1), we have $a_{i} a_{j}^{\prime}=0$ for all $i, j$. Thus $\left(\sum_{i=1}^{n} a_{i} g_{i}\right)\left(\sum_{j=1}^{n} a_{j}^{\prime} h_{j}\right)=0$. Then we have

$$
\left(\sum_{i=1}^{n} a_{i} g_{i}\right) R[M]\left(\sum_{j=1}^{n} a_{j}^{\prime} h_{j}\right)=0
$$

since $R$ is strongly $M$-semicommutative. If we multiply Eq. (2) on the right side by $\left(\sum_{j=1}^{m} a_{j}^{\prime} h_{j}\right)$, then

$0=\left(\left(\sum_{i=1}^{n} a_{i} g_{i}\right)\left(\sum_{j=1}^{m} b_{j}^{\prime} h_{j}\right)+\left(\sum_{i=1}^{n} b_{i} g_{i}\right)\left(\sum_{j=1}^{m} a_{j}^{\prime} h_{j}\right)\right)\left(\sum_{j=1}^{m} a_{j}^{\prime} h_{j}\right)=\left(\sum_{i=1}^{n} b_{i} g_{i}\right)\left(\sum_{j=1}^{m} a_{j}^{\prime} h_{j}\right)^{2}$,

and so $\left(\sum_{i=1}^{n} b_{i} g_{i}\right)\left(\sum_{j=1}^{m} a_{j}^{\prime} h_{j}\right)=0$ for all $i, j$ since $R[M]$ is reduced by Lemma 2.15 and $\left(\sum_{i=1}^{n} a_{i} g_{i}\right)\left(\sum_{j=1}^{m} b_{j}^{\prime} h_{j}\right)=0$. Similarly, from Eq. (4), we have

$$
\left(\sum_{i=1}^{n} d_{i} g_{i}\right)\left(\sum_{j=1}^{m} a_{j}^{\prime} h_{j}\right)=0
$$

and

$$
\left(\sum_{i=1}^{n} a_{i} g_{i}\right)\left(\sum_{j=1}^{m} \dot{d_{j}} h_{j}\right)=0
$$

for all $i, j$. 
Also, if we multiply the Eq. (3), on the right side by $\sum_{j=1}^{m} a_{j}^{\prime} h_{j}$ then

$$
\begin{array}{r}
0=\left(\left(\sum_{i=1}^{n} a_{i} g_{i}\right)\left(\sum_{j=1}^{m} c_{j}^{\prime} h_{j}\right)+\left(\sum_{i=1}^{n} b_{i} g_{i}\right)\left(\sum_{j=1}^{m} d_{j}^{\prime} h_{j}\right)+\left(\sum_{i=1}^{n} c_{i} g_{i}\right)\left(\sum_{j=1}^{m} a_{j}^{\prime} h_{j}\right)\right)\left(\sum_{j=1}^{m} a_{j}^{\prime} h_{j}\right) \\
=\left(\sum_{i=1}^{n} c_{i} g_{i}\right)\left(\sum_{j=1}^{m} a_{j}^{\prime} h_{j}\right)^{2}
\end{array}
$$

implies $\left(\sum_{i=1}^{n} c_{i} g_{i}\right)\left(\sum_{j=1}^{m} a_{j}^{\prime} h_{j}\right)=0$ for all $i, j$ since $R[M]$ is reduced by Lemma 2.15 and

$$
\left(\sum_{i=1}^{n} a_{i} g_{i}\right)\left(\sum_{j=1}^{m} c_{j} h_{j}\right)+\left(\sum_{i=1}^{n} b_{i} g_{i}\right)\left(\sum_{j=1}^{m} \dot{d}_{j} h_{j}\right)=0 .
$$

Multiplying Eq. (5) on the left side by

$$
\left(\sum_{i=1}^{n} a_{i} g_{i}\right)
$$

then

$$
\left(\sum_{i=1}^{n} a_{i} g_{i}\right)\left(\left(\sum_{i=1}^{n} a_{i} g_{i}\right)\left(\sum_{j=1}^{m} c_{j} h_{j}\right)+\left(\sum_{i=1}^{n} b_{i} g_{i}\right)\left(\sum_{j=1}^{m} \dot{d}_{j} h_{j}\right)\right)=\left(\sum_{i=1}^{n} a_{i} g_{i}\right)^{2}\left(\sum_{j=1}^{m} c_{j} h_{j}\right),
$$

and so

$$
\left(\sum_{i=1}^{n} a_{i} g_{i}\right)\left(\sum_{j=1}^{m} c_{j} h_{j}\right)=0
$$

for all $i, j$ since $R[M]$ is reduced by Lemma 2.15 and $\left(\sum_{i=1}^{n} b_{i} g_{i}\right)\left(\sum_{j=1}^{m} d_{j}^{\prime} h_{j}\right)=0$ for all $i, j$. Hence, these yields that

$$
\begin{aligned}
& \left(\sum_{i=1}^{n} b_{i} g_{i}\right) R[M]\left(\sum_{j=1}^{m} a_{j}^{\prime} h_{j}\right)=0, \\
& \left(\sum_{i=1}^{n} a_{i} g_{i}\right) R[M]\left(\sum_{j=1}^{m} \dot{b}_{j}^{\prime} h_{j}\right)=0, \\
& \left(\sum_{i=1}^{n} d_{i} g_{i}\right) R[M]\left(\sum_{j=1}^{m} a_{j}^{\prime} h_{j}\right)=0, \\
& \left(\sum_{i=1}^{n} a_{i} g_{i}\right) R[M]\left(\sum_{j=1}^{m} \dot{d}_{j} h_{j}\right)=0,
\end{aligned}
$$




$$
\begin{aligned}
& \left(\sum_{i=1}^{n} c_{i} g_{i}\right) R[M]\left(\sum_{j=1}^{m}\right. \\
\left.a_{j}^{\prime} h_{j}\right)=0, & \left(\sum_{i=1}^{n} a_{i} g_{i}\right) R[M]\left(\sum_{j=1}^{m} c_{j}^{\prime} h_{j}\right)=0
\end{aligned}
$$

and

$$
\left(\sum_{i=1}^{n} b_{i} g_{i}\right) R[M]\left(\sum_{j=1}^{m} \dot{d}_{j} h_{j}\right)=0
$$

since $R$ is strongly $M$-semicommutative.

Thus

$$
\begin{aligned}
&\left(\begin{array}{ccc}
\sum_{i=1}^{n} a_{i} g_{i} & \sum_{i=1}^{n} b_{i} g_{i} & \sum_{i=1}^{n} c_{i} g_{i} \\
0 & \sum_{i=1}^{n} a_{i} g_{i} & \sum_{i=1}^{n} d_{i} g_{i} \\
0 & 0 & \sum_{i=1}^{n} a_{i} g_{i}
\end{array}\right)\left(\begin{array}{ccc}
r l & t l & s l \\
0 & r l & u l \\
0 & 0 & r l
\end{array}\right) \\
&\left(\begin{array}{ccc}
\sum_{j=1}^{m} a_{j}^{\prime} h_{j} & \sum_{j=1}^{m} b_{j}^{\prime} h_{j} & \sum_{j=1}^{m} c_{j}^{\prime} h_{j} \\
0 & \sum_{j=1}^{m} a_{j} h_{j} & \sum_{j=1}^{m} d_{j} h_{j} \\
0 & 0 & \sum_{j=1}^{m} a_{j} h_{j}
\end{array}\right)=0
\end{aligned}
$$

for any $\gamma=C l \in S_{3}(R)[M]$ where $l \in M$, and

$$
C=\left(\begin{array}{lll}
r & t & s \\
0 & r & u \\
0 & 0 & r
\end{array}\right) \in S_{3}(R),
$$

and so $\alpha S_{3}(R)[M] \beta=0$. Therefore $S_{3}(R)$ is strongly $M$-semicommutative.

Let $R$ be a ring and let

$$
S_{n}(R)=\left\{\left(\begin{array}{ccccc}
a & a_{12} & a_{13} & \cdots & a_{1 n} \\
0 & a & a_{23} & \cdots & a_{2 n} \\
0 & 0 & a & \cdots & a_{3 n} \\
\cdots & \cdots & \cdots & \cdots & \cdots \\
0 & 0 & 0 & 0 & a
\end{array}\right) \mid a, a_{i j} \in R\right\}
$$

where $n$ is a positive integer $\geq 2$. Based on Proposition 2.17, one may suspect that $S_{n}$ may also be strongly $M$-semicommutative for $n \geq 4$. But the following example eliminates the possibility. 
Example 2.18. Let $M$ be a strictly totally ordered monoid and $R$ a reduced ring. Take $e \neq g \in M$. Let $\alpha=e_{12} e+\left(e_{12}-e_{13}\right) g$ and $\beta=e_{34} e+$ $\left(e_{24}+e_{34}\right) g$ be in $S_{n}(R)[M]$ where $e_{i j}$ 's are the matrix units in $S_{n}(R)(n \geq 4)$. Then $\alpha \beta=0$, but $\alpha \gamma \beta \neq 0$, where $\gamma=e_{13} e+\left(e_{13}+e_{23}\right) g \in S_{n}(R)[M]$. Thus $S_{n}(R)[M]$ is not strongly $M$-semicommutative $(n \geq 4)$.

The converse of Proposition 2.4 is not true in general by Proposition 2.17 as follows:

Example 2.19. Let $M$ be a strictly totally ordered monoid and $R$ a reduced ring. By Proposition $2.17 S_{3}(R)$ is strongly $M$-smeicommutative. However, $\alpha \beta=0$, where $\alpha=e_{13} e+e_{23} g, \beta=e_{12} e+e_{13} g \in S_{3}(R)[M]$, with $e \neq g \in M$. But $\beta \alpha \neq 0$. So $S_{3}(R)$ is not strongly $M$-reversible.

Given a ring $\mathrm{R}$ and a bimodule ${ }_{R} M_{R}$, the trivial extension of $R$ by $M$ is the ring $T(R, M)=R \bigoplus M$ with the usual addition and the following multiplication $\left(r_{1}, m_{1}\right)\left(r_{2}, m_{2}\right)=\left(r_{1} r_{2}, r_{1} m_{2}+m_{1} r_{2}\right)$. This is isomorphic to the ring of all matrices $\left(\begin{array}{cc}r & m \\ 0 & r\end{array}\right)$, where $r \in R, m \in M$ and the usual matrix operations are used.

Proposition 2.20. Let $M$ be a strictly totally ordered monoid and $R$ a reduced ring. If $R$ is strongly $M$-smeicommutative, then $T(R, R)$ is strongly $M$-smeicommutative.

Proof. Note that $T(R, R)$ is isomorphic to the ring

$$
\left\{\left(\begin{array}{lll}
a & b & 0 \\
0 & a & 0 \\
0 & 0 & a
\end{array}\right)^{\mid a, b \in R}\right\} .
$$

Now the result follows from Proposition 2.17 and from the fact that every subring of strongly $M$-smeicommutative ring is also strongly $M$-smeicommutative.

\section{Acknowledgments}

We would like to thank Professor Liu Zhongkui for his valuable comments and we would like to thank the managements of University of Khartoum and Northwest Normal University. Also the authors thank the referee for his/her a very careful reading of the paper. 


\section{References}

[1] A. B. Singh, P. Juyal and M. R. Khan, Strongly reversible relative to monoid, Int. J. pure and appl. Math., 63, No. 1 (2010), 1-7.

[2] C. Huh, Y. Lee, A. Smoktunowicz, Armendariz rings and semicommutative rings, Comm. Algebra, 30, No. 2 (2002), 751-761.

[3] D. D. Anderson, V. Camillo, Semigroups and rings whose zero products commute, Comm. Algebra, 27, No. 6 (1999), 2847-2852.

[4] D. S. Passman, The Algebraic Structure of Group Rings, John Wiley, New York, (1977).

[5] G. F. Birkenmeier, J.K. Park, Triangular matrix representations of ring extensions, J. Algebra, 265, (2003), 457-477.

[6] G. Y. Shin, Prime ideals and sheaf representation of a pseudo symmetric ring, Trans. Amer. Math. Soc., 184, (1973), 43-60.

[7] G. Yang, Z. Liu, On strongly reversible rings, Taiwanese J. Math., 12, No. 1 (2008), 129-136.

[8] G. Yang, Semicommutative and Reduced Rings, Vietnam J. Math., 35, No. 3 (2007), 309-315.

[9] J. Lambek, On the representation of modules by sheaves of factor modules, Canad. Math. Bull., 14, No. 3 (1971), 359-368.

[10] J. M. Habeb, A note on zero commutative and duo rings, Math. J. Okayama Univ., 32, (1990), 73-76.

[11] M. B. Rege, S. Chhawchharia, Armendariz rings, Proc. Japan Acad. Ser. A math. Sci., 73 (1997), 14-17.

[12] N. K. Kim, Y. Lee, Extension of reversible rings, J. Pure Appl. Algebra, 185, (2003), 207-223.

[13] N. K. Kim, Y. Lee, Armendariz rings and reduced rings, J. Algebra, 223 (2000), 477-488.

[14] P. M. Cohn, Reversible rings, Bull. London Math. Soc., 31, (1999), 641648. 
[15] P. P. Nielsen, Semicommutativity and the McCoy condition, J. Algebra, 298, (2006), 134-141.

[16] T. K. Kwak, Y. Lee and S. J. Yun, The Armendariz property on ideals, J. Algebra, 354, (2012), 121-135.

[17] Y. Gang and D.R. Juan, Rings over which polynomial rings are semicommutative, Vietnam J. Math., 37, No. 4 (2009), 527-535.

[18] Y. Hirano, On annihilator ideals of a polynomial ring over a noncommutative ring, J. Pure Appl. Algebra, 168, (2002) 45-52.

[19] Z. Liu, Armendariz rings relative to a monoid, Comm. Algebra, 33, No. 3 (2005), 649-661.

[20] Z. Liu, Semicommutative Subrings of Matrix Rings, J. Math. Reseach and Exposition, 26, No. 2 (2006), 264-268. 